\title{
CHARACTERIZATION OF THE RAT MUTANT DYSTONIC (dt): A NEW ANIMAL MODEL OF DYSTONIA MUSCULORUM DEFORMANS ${ }^{1}$
}

\author{
JOAN F. LORDEN, ${ }^{2}$ TINA WILLIAMS MCKEON, $\ddagger$ HENRY J. BAKER, NANCY COX, AND \\ STEVEN U. WALKLEY ${ }^{3}$
}

\author{
Departments of *Psychology, $\ddagger$ Anatomy, and Comparative Medicine, University of Alabama in Birmingham, \\ Birmingham, Alabama 35294
}

Received October 26, 1982; Revised March 19, 1984; Accepted March 20, 1984

\begin{abstract}
An inherited neurological disorder characterized by sustaincd twisting movements during waking has been discovered in Sprague-Dawley rats. The mutation follows an autosomal recessive pattern of inheritance and has been named dystonic $(d t)$. The rat mutants are indistinguishable from normal littermates in open field behavior and climbing activity prior to postnatal days 9 to 10 . Clinical signs begin to appear on day 10 and include twisting of the axial musculature, hyperflexion of the trunk, self-clasping of forelimbs and hindlimbs, and poor placement of the limbs during locomotion. No morphological lesions of neural or non-neural tissues have been observed with routine light microscopy. Dystonic rats demonstrate significantly elevated cerebellar norepinephrine levels, although levels in other terminal fields of the locus ceruleus are similar to those of normal littermates. No differences in the pattern or density of noradrenergic innervation were apparent in cerebellar tissue from $d t$ rats examined with histochemical fluorescence techniques. These mutants were less sensitive than unaffected littermates to the akinesic effects of the dopamine blocker haloperidol. However, striatal dopamine levels were not reliably different from normal in $d t$ rats, and their response to the movementstimulating effects of apomorphine appeared normal. These findings suggest the presence of biochemical disturbances in the extrapyramidal system of $d t$ rats. The $d t$ rat may provide a useful model for human dystonia musculorum deformans.
\end{abstract}

Sustained, involuntary twisting movements are characteristic of a variety of dyskinetic illnesses referred to as dystonias (Marsden, 1980). Dystonic symptoms accompany a number of disease states, including well known degenerative conditions, such as Huntington's chorea and Parkinson's disease, and a variety of inflammatory, cerebrovascular, iatrogenic, and metabolic disorders (Zeman, 1970). Among the array of clinically evident dystonic states are the hereditary torsion dystonias (dystonia musculorum deformans) (Fahn and Eldridge, 1976). Autosomal recessive and autosomal dominant forms are established disease entities in human populations. However, no convincing pathological change has been identified in post mortem studies of brains from patients dying with the disease (Eldridge, 1970; Zeman, 1970).

The absence of demonstrable morphological lesions at the light microscopic level and the finding that dystonic movements may occur as a complication of $L$-dopa therapy in patients with Parkinson's disease (Marsden and Harrison, 1974; Marsden, 1976; Zeman, 1976) have encouraged speculation that a bio-

\footnotetext{
${ }^{1}$ This work was supported by Grant NS18062 from the National Institute of Neurological and Communicative Disorders and Stroke. We thank Mary Martin, Leigh Lindsey, Nancy Haskew, and Linda Hayley for their assistance in maintaining the colony and collecting data. We are also indebted to Dr. Lawrence E. Mays for the design of both hardware and software for the activity monitors.

${ }^{2}$ To whom correspondence should be addressed.

${ }^{3}$ Present address: Department of Neuroscience, Albert Einstein College of Medicine, Bronx, NY 10462.
}

chemical disturbance may be the cause of the disease. Abnormalities in catecholamine metabolism have been suggested on the basis of some limited clinical data. Specifically, increased activity of plasma dopamine $\beta$-hydroxylase (Askenasy et al., 1980; Wooten et al., 1973), elevation of plasma norepinephrine (Zeigler, 1976), and decreased ventricular fluid levels of the norepinephrine metabolite, 3-methoxy-4-hydroxyphenylglycol (MHPG) (Wolfson et al., 1983), have been reported. There is also some evidence that patients with adult- but not childhoodonset dystonia may be distinguished by decreased cerebrospinal levels of the dopamine metabolite homovanillic acid (Tabaddor et al., 1978).

Investigations of animal models of torsion dystonia have been confined primarily to the dystonia musculorum (dt) mouse, an autosomal recessive mutation originally described by Duchen and his co-workers (1964). Numerous lesions have been identified in the $d t$ mouse. These include peripheral nervous system and spinal cord lesions, such as axonal swellings, gliotic changes, and Wallerian degeneration (Duchen et al., 1964). Morphological changes have also been noted in the red nucleus and caudate (Messer and Strominger, 1980). Biochemical measurements have yielded additional evidence of central nervous system pathology in the mouse mutant. Messer and Gordon (1979) have reported data suggesting the presence of an endogenous inhibitor of glutamate uptake in the basal ganglia, and evidence of altered cerebellar noradrenergic metabolism has been found (Riker et al., 1981). It appears that exploration of the full extent of lesions in the mouse model of dystonia has only begun. However, because of the presence of 
significant peripheral nervous system and spinal cord lesions which have not been observed in humans suffering from dystonia, the nature of the human and murine diseases may be dissimilar.

We have recently discovered a mutation in Sprague-Dawley rats with the clinical characteristics of torsion dystonia. This mutant, named dystonic ( $d t$ ), is distinguished from the $d t$ mouse by the absence of obvious central and peripheral neuropathological lesions. The data presented here describe the behavioral characteristics of the mutants, the pattern of inheritance, and the development of the $d t$ syndrome. We also report the results of the anatomical, pharmacological, and neurochemical investigations with which we have endeavored to assess the potential value of this mutant as an animal model of human torsion dystonia. Regional measurements of central catecholamines were undertaken, and the behavioral effects of dopamine agonists and antagonists were examined.

\section{Materials and Methods}

Animals. Mutant and phenotypically normal rats were obtained from the dystonic colony maintained at the University of Alabama in Birmingham. Mutants were obtained by mating heterozygotes. Pups born in the dystonic colony were routinely weighed on postnatal days 8,10 , 12 , and 16 as a general index of health and to assess the ability of the mutants to compete with normal littermates for nutrition. From day 16 until sacrifice, dystonic rats received supplemental hand feedings of wet mash made from standard laboratory chow or Esbilac (Borden), a milk-based diet. Nine litters of 8 to 13 pups each were used for statistical analysis of the growth curves of rats from the dystonic colony. All the litters contained dystonic pups. A total of 17 male and 18 female dystonic rats and 28 male and 34 female normal rats were measured.

Pedigree analysis. Seventy-six litters from the dystonic colony were analyzed over a 3-year period. Litters selected for analysis were from parents known to have produced affected young, but not all of these litters included mutant pups.

Behavioral observations. Pups of unknown phenotype were videotaped individually in an open field beginning on postnatal day 6 to determine the earliest age at which a reliable phenotypic differentiation of normal and mutant pups was possible. Each pup was videotaped daily for 1 to $2 \mathrm{~min}$ on postnatal days 6 to 12 . Videotapes were reviewed to assess whether qualitative behavioral differences existed between normal and $d t$ rats prior to the onset of obvious clinical symptomatology.

A climbing test similar to that described by Altman and Sudarshan (1975) was also used to evaluate the locomotor development of $d t$ pups. A $1-\mathrm{cm}$ wire mesh incline was placed at $45,60,70$, or $80^{\circ}$ depending on the age of the pups. Pups of 6 to 12 days of age were placed head up on the incline. A positive score was recorded if both hindlimbs moved upward at least $2 \mathrm{~cm}$. Each pup was given three trials in which to display a positive response. At least 10 mutants and 20 normal littermates were tested on the developmentally appropriate inclines on each day.

Anatomical investigations. Because $d t$ rats have greater difficulty feeding than do normal rats, whole brain weights were measured in affected and unaffected pups as an index of brain development. Rats were decapitated and the brains removed and weighed at 16,18 , and 20 days of age. At least eight $d t$ and eight phenotypically normal littermates were used at each age. Gross examination of brain conformation and routine light microscopy studies were performed using four mutants and four normal littermates 12 to 20 days of age. The rats were anesthetized and perfused intracardially with $10 \%$ buffered formalin. Neural and non-neural tissues were paraffin embedded, sectioned, and stained with hematoxylin and eosin. Some sections of neural tissue were stained with luxol fast blue or periodic acid-Schiff reagent. Particular attention was directed toward examination of the spinal cord for gliotic and Wallerian degenerative changes. An additional four mutant and four normal rats of 21 to 25 days of age were anesthetized and perfused as described above. The brains were sectioned at $48 \mu \mathrm{m}$ thickness on a cryotome and stained with cresyl violet. The striatum and red nucleus were examined in detail for morphological changes such as those reported in the mouse dystonia musculorum by Messer and Strominger (1980).

Since hyperinnervation of the cerebellum by the noradrenergic projection of the locus ceruleus has been reported in several mutants with motor disorders (Landis et al., 1975; Levitt and Noebels, 1981; Kos- trzewa et al., 1982; Muramoto et al., 1982), the cerebella from four $d t$ and four normal rats were prepared for histofluorescent visualization of monoamines. Twenty-day-old rats were injected intraperitoneally with $200 \mathrm{mg} / \mathrm{kg}$ of pargyline and decapitated $1 \mathrm{hr}$ later. The brains were rapidly removed and sagittally sectioned at $30 \mu \mathrm{m}$ in a cyrotome maintained at $-30^{\circ} \mathrm{C}$. Sections were processed using the sucrosepotassium phosphate-glyoxylic acid method described by de la Torre (1980) and examined with a Leitz Orthoplan microscope equipped for epifluorescence. Adjacent sections were mounted and stained with cresyl violet. Two additional animals from each group were examined without pargyline pretreatment. For comparisons between $d t$ and normal rats, both midvermian and paravermian sections were examined. The continuity of the superior cerehellar peduncle with the cerebellar medulla served as a landmark for the paravermian sections. The sections were divided into quadrants, and areas of high, moderate, and low fluorescent intensity were examined and photographed in each quadrant.

Neurochemical analysis. Regional assays of norepinephrine (NE) and dopamine (DA) were performed in both motor and nonmotor areas in 10 mutants and 10 normal littermates between 16 and 25 days of age. After decapitation, the cerebellum, striatum, and hippocampus were dissected and frozen in liquid nitrogen as described by Morley and coworkers (1977). The telencephalic tissue remaining after this dissection was also frozen and assayed. Fluorometric assays were performed using a modification of the technique described by Jacobowitz and Richardson (1978).

Psychopharmacological studies. The effects of haloperidol (Haldol, $\mathrm{McNeil),} \mathrm{a} \mathrm{DA} \mathrm{receptor} \mathrm{blocker,} \mathrm{and} \mathrm{apomorphine} \mathrm{(apomorphine} \mathrm{HCl}$, Sigma), a DA agonist, were evaluated on the movements of dystonic rats between 16 and 25 days of age. To determine whether there were any drug effects specific to dystonic movements and postures, mutants were videotaped for $3 \mathrm{~min}$ in an open field before and after drug administration. The postdrug test was conducted $30 \mathrm{~min}$ after injection for animals treated with apomorphine $(1,2$, or $3 \mathrm{mg} / \mathrm{kg})$ and $60 \mathrm{~min}$ after for those treated with haloperidol $(10 \mathrm{mg} / \mathrm{kg})$. All drugs were administered intraperitoneally. During these periods mutants were scored for characteristic movements or postures: torticollis; falls (movements involving the axial musculature and resulting in a fall to the side with the extremities rigidly extended); paw clasps (front paws, hind paws, or one front and one hind paw clasped together); runs (rapid forward movements in which left and right limbs are moved simultaneously). Each occurrence of these events was counted.

In addition to the videotaped trials, the locomotor activity of mutants and normal littermates was measured in automatic activity monitors after intraperitoneal injections of haloperidol or apomorphine. Activity following drug treatment was compared with activity following $0.9 \%$ saline injections in 18- to 20-day-old pups. At this age normal rats display catalepsy in response to neuroleptics (Baez et al., 1976) and increases in general motor activity in response to apomorphine (Lipton et al., 1980; Reinstein et al., 1978). Animals received drug or saline injections on successive days. The order of treatment was counterbalanced within each group.

The activity monitors were chambers measuring $18 \times 28 \mathrm{~cm}$ and crossed by eight infrared beams positioned $3.25 \mathrm{~cm}$ apart on the long wall. For testing, rats were housed individually in standard clear plastic rodent cages that were placed in the activity chambers. The infrared beams crossed the cages $2 \mathrm{~cm}$ above the floor of the cages. The cage floors were covered with bedding material and warmed to $28^{\circ} \mathrm{C}$. Breaks in the infrared beams were detected by phototransistors and counted by an Apple II computer. Rats were placed in the activity monitoring chambers and allowed $30 \mathrm{~min}$ to adapt to the environment prior to drug or saline injection. Immediately following the injections, the rats were returned to the chambers and activity was measured for $30 \mathrm{~min}$ after apomorphine injections of either $0.5 \mathrm{mg} / \mathrm{kg}$ or $1 \mathrm{mg} / \mathrm{kg}$ and for 60 min after haloperidol injections of $10 \mathrm{mg} / \mathrm{kg}$. All rats were tested between 2 and 5 P.M., and both $d t$ and normal rats were tested at the same time. Each rat received only one drug and dose. Beam crossings were summed over 5-min periods, and average activity scores were obtained for each session based on the 5 -min counts. The data for the apomorphine and haloperidol tests were analyzed in separate repeated measures analyses of variance.

\section{Results}

Clinical syndrome. Dystonic rats appear normal until 10 days after birth, when a stiff paddling gait with frequent falling to 
the side, excessive pivoting or circling, and torticollis (twisting of the neck, frequently from side to side) are exhibited in the waking state. The disorder progresses rapidly over the next few days. Advanced clinical signs include abnormal limb placement during locomotion, falling to the side, turning in of the forelimbs, self-clasping of forelimbs and hindlimbs, rigidity of the tail, and hyperflexion of the trunk (Fig. 1). There is resistance to passive movements of the limbs during the dystonic spasms. The limbs spring back to their original position, if displaced by the observer.

The growth curves of normal and dystonic rats were analyzed in a phenotype $\times$ age repeated measures analysis of variance followed by Newman-Keuls tests. Separate statistical analyses were carried out for male and female rats. Body weights of normal and dystonic rats were similar at least until postnatal day 16 , when normal pups began to show evidence of more successful competition for nutrition than mutants, as indicated by a slightly higher mean body weight. Sixteen-day-old male but not female mutants weighed significantly $(p<0.05)$ less than normal pups of the same age and sex. By day 16 the mean body weight for normal male rats was $25.3 \mathrm{gm}(\mathrm{SD}=4.0)$ and for dystonic males, $22.2 \pm 2.8 \mathrm{gm}$. For female rats mean body weights on day 16 were $24.2 \pm 4.4$ gm for normal rats and 21.9 $\pm 4.8 \mathrm{gm}$ for mutants. The weight differences between affected and unaffected pups are exaggerated after weaning, although the mutants can be maintained in apparent good health for at least 30 to 35 days with hand feeding.

Pedigree analysis. A partial pedigree of a rat family with dystonia is shown in Figure 2. Of 670 progeny included in the pedigree analysis, 170 had neurological signs and 509 were phenotypically normal. The ratio of diseased pups to total pups (1:4) is that expected for an autosomal recessive trait. Males and females were affected equally, and phenotypic expression of the mutation occurred only in homozygotes.

Open field analysis. Prior to day 10 , dystonic pups displayed motor activities in the open field that were qualitatively similar to those of normal littermates. The activities evaluated included head elevation, grooming, pivoting, crawling, and stability in the quadruped stance. On day 10 , when normal littermates began coordinated crawling, dystonic pups showed only a few asymptomatic steps with their movement dominated by pivoting and frequent falling to the side. At 11 days of age, normal pups showed more coordinated crawling, a decline in pivoting behavior, and only a rare loss of balance from a quadruped stance. In 11-day-old dystonic pups, pivoting behavior increased and ability to maintain a quadruped stance was limited. Torticollis, alternating irregularly from side to side, and frequent falling were apparent at this age. By 12 days of age, dystonic pups are readily differentiated from normal littermates even by an untrained observer.

Climbing tests. No reliable difference in climbing ability between normal and dystonic pups was evident when pups were tested on $45^{\circ}$ or $60^{\circ}$ inclines through day 10 . Significant differences in climbing ability began to emerge in 9- and 10-day-old pups on $70^{\circ}$ and $80^{\circ}$ inclines (Fig. 3). Normal pups showed significantly more climbing ability than dystonic pups at 9 days of age on the $70^{\circ}$ incline and at 10,11 , and 12 days on both the $70^{\circ}$ and $80^{\circ}$ inclines.
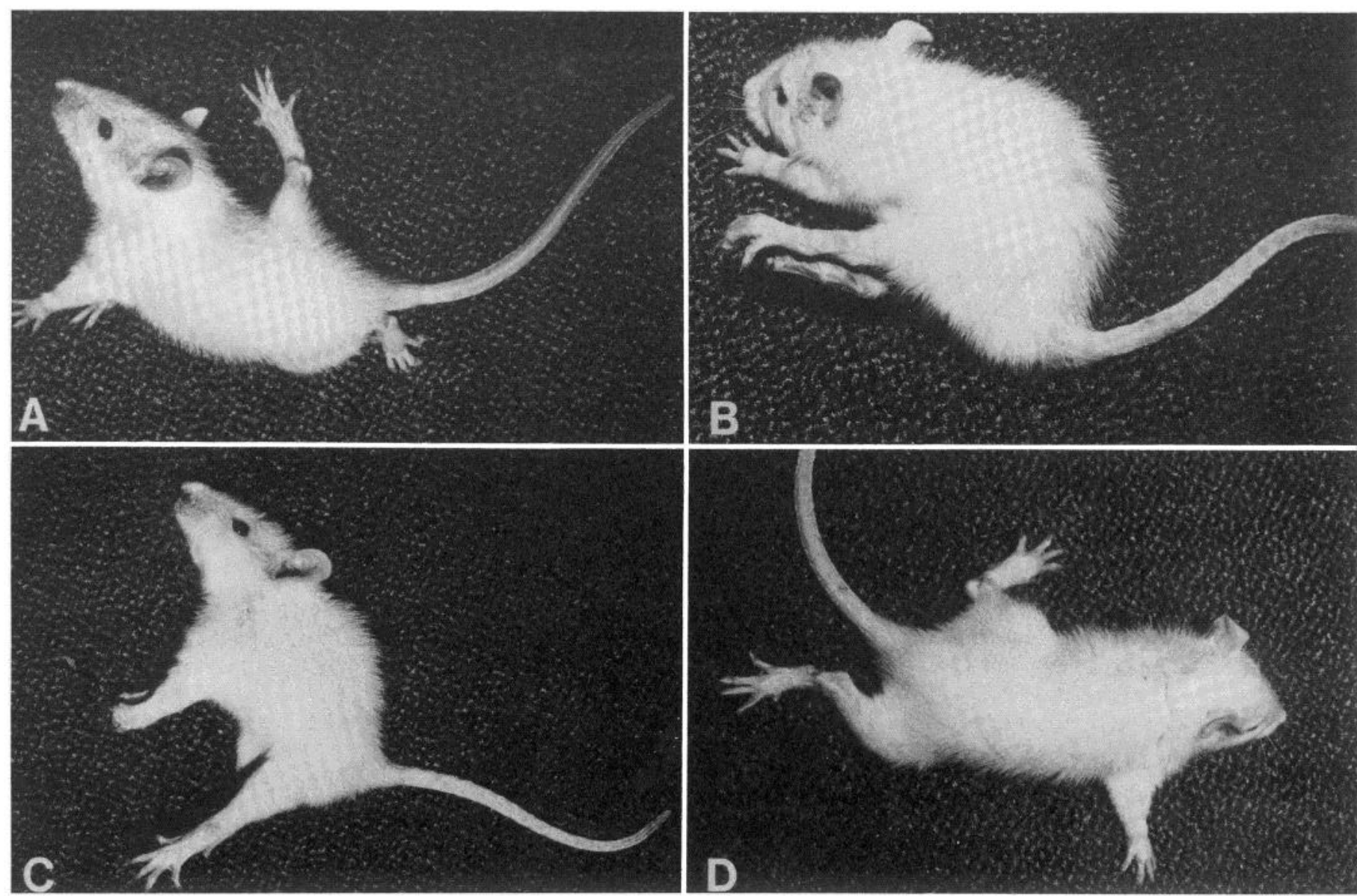

Figure 1. Advanced clinical signs of the dystonic $(d t)$ rat. $A$, Torticollis. $B$, Hindpaw clasp as the animal begins to roll from right to left and hyperflexion of the trunk. $C$, Forepaw clasp and rigid extension of the limbs. $D$, Poor limb placement during locomotion. 


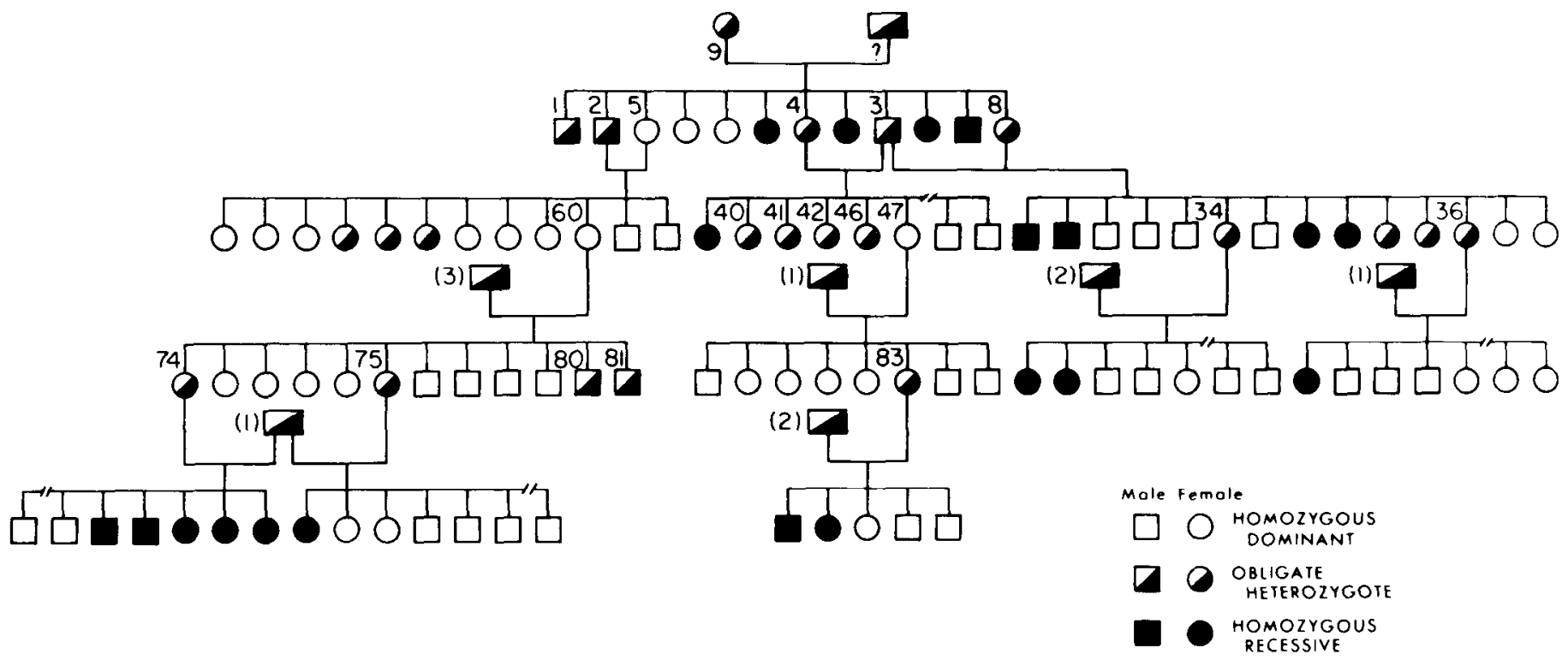

Figure 2. Partial pedigree of a rat family with dystonia showing selected sibships. Recessive rats are defined by neurological signs. All rats shown to be obligate heterozygotes are parents of affected pups. Females 5,47 , and 60 are proven homozygous dominant by progeny testing. The remainder of the phenotypically normal individuals may be of dominant or heterozygous genotype. Broken lines indicate that the sibship is larger than that shown.

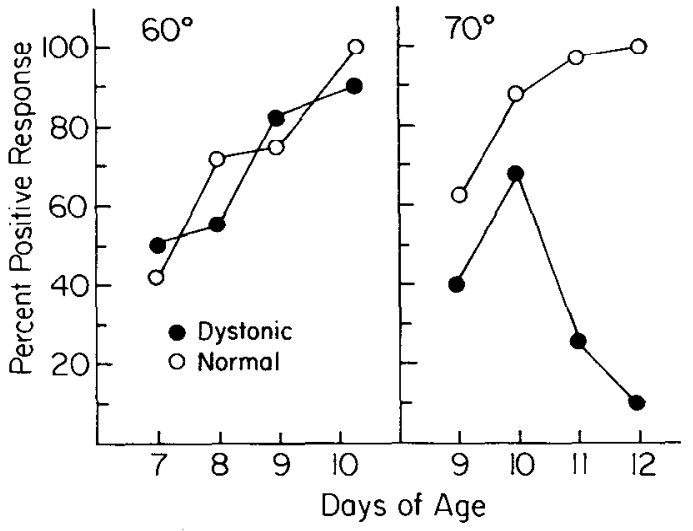

Figure 3. Climbing ability of normal and dystonic rats 7 to 12 days of age. Animals were placed on a wire mesh incline and given a positive or negative score for climbing ability. Results are indicated as the percentage of animals in each group ( $n=$ at least 5) showing a positive response. Normal and dystonic rats are equally capable of climbing a $60^{\circ}$ incline through day 10 . Normal rats show more climbing ability than 9 - to 12-day-old dystonic rats on a $70^{\circ}$ incline. Data were analyzed by the $\chi^{2}$ test.

Anatomical investigations. Mean brain weights were identical in 16-day-old $d t$ and normal rats ( $n=14 /$ group) but differed significantly in older rats, when analyzed in a phenotype $\times$ age analysis of variance followed by Newman-Keuls tests. Brain weights in normal rats increased by $14 \%$ over the interval from postnatal days 16 to 20 . However, dystonic rats had similar brain weights at 16,18 , and 20 days of age. The mean brain weight $( \pm$ SD) of the normal pups on day 20 was $1.39 \pm 0.05$ gm and of the mutants, $1.24 \pm 0.07 \mathrm{gm}$ ( $n=9 /$ group).

Gross examination of brain conformation revealed no difference between normal and dystonic rats. Light microscopic examination of neural and non-neural tissue also failed to reveal significant morphological differences. Specifically, the $d t$ rats showed none of the gliotic or Wallerian degenerative changes seen in the spinal cord or peripheral nervous system of the $d t$ mouse (Duchen et al., 1964), nor were the degenerative changes that have been observed in the red nucleus or striatum (Messer and Strominger, 1980) of the $d t$ mouse in evidence.
Examination of the noradrenergic fibers of the cerebellum in $d t$ rats revealed a pattern of innervation similar to that seen in normal rats (Fig. 4). Fluorescent varicosities were evident in all cerebellar layers, although the highest concentration was found in the Purkinje cell layer. Normal and $d t$ rat cerebella could not be discriminated on the basis of density of fluorescent terminals. However, the intensity of fluorescence appeared to be increased in the mutants.

Neurochemical analysis. A significant elevation of NE (42\%) was found in the cerebellum of the mutant rats in comparison with normal littermates (Table I). However, no reliable difference was evident in the hippocampus or the remaining telencephalon. Nor were any significant differences apparent in DA levels of normal and mutant rats in either the striatum or the remaining telencephalon. Tissue section weights did not differ reliably in mutant and normal rats in this sample.

Psychopharmacological studies. Mutant pups showed no inhibition of dystonic movements and postures in 3-min open field tests following a $10 \mathrm{mg} / \mathrm{kg}$ dose of haloperidol. The mean number of dystonic movements ( \pm SE) counted before and after haloperidol administration were $46.2 \pm 5.1$ and $44.2 \pm$ 16.2 , respectively, $(n=4)$. The only reliable change observed in a specific behavior characteristic of the $d t$ rat was an increase in the number of "runs" following haloperidol injection. This behavior increased from a mean frequency (number per 3-min period $\pm \mathrm{SE}$ ) of $4.8 \pm 2.1$ to $11.0 \pm 1.6$ (paired $t=9.99, d f=3$, $p<0.01$ ). In the 3 -min open field observation, apomorphine did not appear to produce any overall effect on the frequency of dystonic movements. Only at the highest dose used ( $3 \mathrm{mg} /$ $\mathrm{kg}$ ) was any statistically significant change in the frequency of a specific movement or posture observed. The mean number of occurrences of torticollis declined from $19.4 \pm 2.8$ (mean $\pm \mathrm{SE}$ ) in the predrug trial to $16.2 \pm 2.0$ in the postdrug trial (paired $t$ $=2.36, d f=4, p<0.05$ ). At this dose the total number of occurrences of dystonic movements was $42.4 \pm 2.6$ (mean \pm $\mathrm{SE}$ ) prior to the drug treatment and $38.4 \pm 3.7$ after. No reliable effects were seen at lower doses of apomorphine.

Observations made on both normal and $d t$ rats in the automatic activity monitors indicated that the activity levels of the two groups were not significantly different during the saline trials. Haloperidol produced akinesia in both groups $(F(1,16)$ 

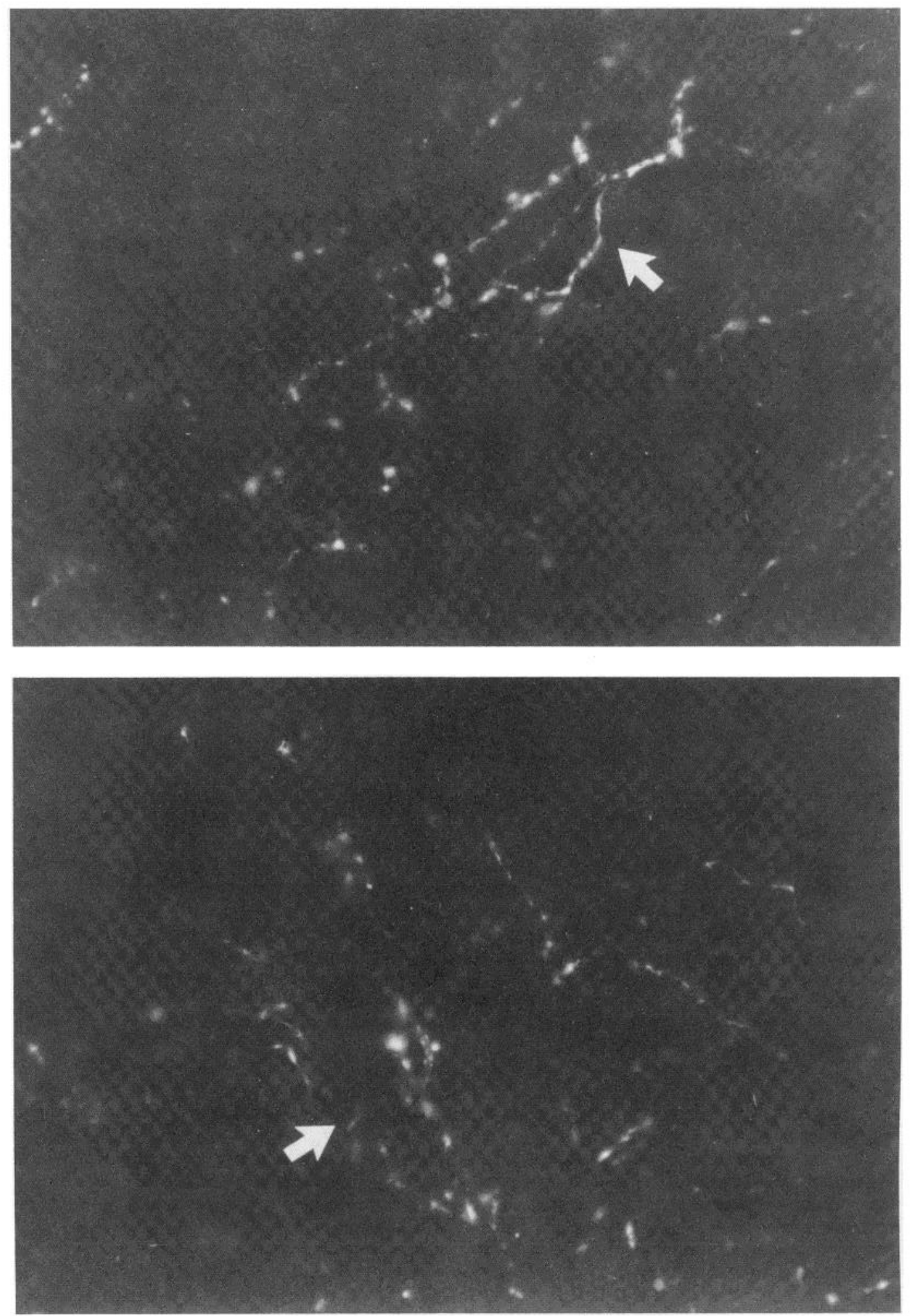

Figure 4. Catecholamine fluorescence in the cerebella of pargyline treated 20-day-old $d t$ (top panel) and normal (bottom) rats. Arrows point to the Purkinje cell layer. Similar patterns of innervation were evident in both types of rats, although fluorescence intensity was typically greater in $d t$ rats.

$=71.0, p<0.0001)$. However, the effect was not as pronounced in the $d t$ animals, as indicated by a significant group $\times$ trial interaction $(F(1,16)=4.49, p<0.05)$. These data are summarized in Table II where activity following drug treatment is expressed as a percentage of the activity that occurred during the saline trials.
Apomorphine increased activity levels in both groups of rats in a dose-dependent manner. However, only the $1 \mathrm{mg} / \mathrm{kg}$ dose of apomorphine increased activity levels significantly above those of saline trials $(F(1,28)=6.17, p<0.02)$ (Table II). There was no indication of a difference in the effect of the drug on the two groups. 
TABLE I

Mean catecholamine levels $( \pm S E)$ in brains of normal and dystonic rats

Values for amine levels are expressed as micrograms of amine per gram of fresh brain. Sample size equals 9 for normal hippocampus and 10 for all other sections. Statistical comparisons were made with the Student's $t$ test.

\begin{tabular}{|c|c|c|c|c|c|c|}
\hline \multirow{2}{*}{ Tissue Section } & \multicolumn{3}{|c|}{ Normal } & \multicolumn{3}{|c|}{ Dystonic } \\
\hline & Section Weight & NE & DA & Section Weight & NE & DA \\
\hline & $g m$ & & & $g m$ & & \\
\hline Cerebellum & $0.139 \pm 0.009$ & $0.175 \pm 0.032$ & & $0.136 \pm 0.006$ & $0.248 \pm 0.028^{a}$ & \\
\hline Striatum & $0.061 \pm 0.004$ & & $5.096 \pm 0.195$ & $0.055 \pm 0.066$ & & $4.832 \pm 0.392$ \\
\hline Hippocampus & $0.088 \pm 0.006$ & $0.270 \pm 0.044$ & & $0.077 \pm 0.003$ & $0.306 \pm 0.027$ & \\
\hline Remaining telencephalon & $0.569 \pm 0.018$ & $0.111 \pm 0.006$ & $0.401 \pm 0.031$ & $0.520 \pm 0.019$ & $0.123 \pm 0.006$ & $0.393 \pm 0.017$ \\
\hline
\end{tabular}

${ }^{a}$ Differs from normal, $p<0.01$.

\section{TABLE II}

Activity levels of dystonic and normal rats following treatment with haloperidol or apomorphine

Activity values are expressed as a mean percentage of saline control trials $( \pm \mathrm{SE})$. Sample sizes equal nine per group for haloperidol and eight per group for each dose of apomorphine. All rats were 18 to 20 days old at the time of testing. The observation periods were $30 \mathrm{~min}$ for apomorphine and $60 \mathrm{~min}$ for haloperidol.

\begin{tabular}{lccc}
\hline & & \multicolumn{2}{c}{ Activity } \\
& Dose & \multicolumn{2}{c}{ (Percentage of Control Trial) } \\
\cline { 2 - 4 } & & Dystonic & Normal \\
\hline & $m g / k g$ & & \\
Haloperidol & 10 & $37.8 \pm 9.9^{a, b}$ & $13.8 \pm 2.5^{b}$ \\
Apomorphine & 0.5 & $132.0 \pm 32.3$ & $118.4 \pm 48.9$ \\
Apomorphine & 1 & $251.4 \pm 37.4^{b}$ & $267.1 \pm 72.0^{b}$
\end{tabular}

${ }^{a}$ Differs from normal rats given the same treatment, $p<0.01$.

${ }^{b}$ Differs from saline control trials, $p<0.05$.

\section{Discussion}

The $d t$ rat displays clinical symptoms of dystonia with an onset at postnatal day 9. The disease follows an autosomal recessive pattern of inheritance and is not associated with any obvious morphological lesion of the central or peripheral nervous system. However, the results of pharmacological and neurochemical studies suggest the presence of abnormalities in the extrapyramidal system.

At the behavioral level, the symptoms seen in the $d t$ rats are similar to those described for humans with dystonia musculorum deformans (Fahn and Eldridge, 1976; Marsden, 1976). The abnormalities seen in the $d t$ rat involve sustained twisting of the limbs or axial musculature, are evident only in the waking state, and occur following a period of normal development. The abnormal postures displayed by the $d t$ rat may be maintained for several seconds, and during this time the muscles resist passive stretch. As in the autosomal recessive form of torsion dystonia, once apparent the clinical symptoms of the $d t$ rat progress rapidly. The dystonic spasms of an affected pup can be seen in the home cage, but the severity and frequency are greatly increased by placing the animal on a smooth surface in the center of a large open area.

Neuroanatomically, the disease of the $d t$ rat shares with dystonia musculorum deformans an absence of any gross morphological abnormality or sign of degenerative processes in either the central or peripheral nervous system. The apparent absence of degenerative changes in the basal ganglia in Nisslstained material from $d t$ rats showing advanced clinical signs is complemented by recent studies of Golgi-impregnated material (McKeon et al., 1984). These studies have also failed to indicate conspicuous cytoarchitectural or morphological changes in the striatum of $d t$ rats. No qualitative differences were detected in the cell types represented, the relative abundance of different cell types, or the dendritic and somatic characteristics of the identified cell types of normal and dystonic rats.

Clearly, more detailed anatomical investigations may reveal morphological disturbances in both $d t$ rats and in humans dying with idiopathic torsion dystonia. However, the light microscopic studies of the nervous system of the $d t$ rat conducted to date serve to distinguish the disease of this mutant from other neurological diseases. For example, there is no evidence in the $d t$ rat of the cerebellar hypoplasia or abnormalities in foliation or cell arrangement found in several other neurological mutants with movement disorders (e.g., Landis et al., 1975). Nor are the central and peripheral nervous system abnormalities seen in Nissl-stained material from the dystonia musculorum mouse in evidence in the $d t$ rat (Duchen et al., 1964; Messer and Strominger, 1980). The apparent morphological integrity of the extrapyramidal system in $d t$ rats with advanced clinical signs also distinguishes the disease of this mutant from degenerative diseases of the basal ganglia, such as Parkinson's disease and Huntington's chorea.

The presence of elevated NE levels in the cerebellum of the $d t$ rat is consistent with evidence of biochemical abnormalities in the noradrenergic systems of humans with torsion dystonia. Specifically, decreased levels of the NE metabolite MHPG have been reported in a subset of patients with dystonia, those displaying a childhood onset and rapid progression of symptoms (Wolfson et al., 1983). The MHPG levels of this group were significantly lower than those of another group with more focal adult-onset dystonia as well as being lower than a group of patients with other neurological disease, including some with motor disorders. Childhood-onset dystonia is generally thought to be inherited (Marsden and Harrison, 1974). Thus, alterations in central noradrenergic metabolism may be of particular significance in the etiology or pathogenesis of inherited dystonia.

Cerebellar NE metabolism may be altered as a compensatory response to another as yet undetermined biochemical abnormality. Elevations in cerebellar NE levels have been induced experimentally in the rat on or before postnatal day 21 with severe undernutrition during the pre- and early postnatal period (Miller et al., 1982). Several lines of evidence suggest that undernutrition is not the cause of the differences we report in the $d t$ rat. First, growth curves for the $d t$ rat appear normal during the early postnatal period. The mutants begin to lag their unaffected littermates only after day 16 , suggesting that their nutrition is adequate through the suckling period. Brain weight follows a pattern similar to body weight. This is not the case in studies of undernutrition in which increases in $\mathrm{NE}$ concentration are correlated with a decrease in brain weight (Shoemaker and Wurtman, 1971; Stern et al., 1975). In addition, elevations in NE levels are widespread in undernourished animals, rather than localized as in the $d t$ rat.

Alterations in cerebellar NE have been reported in numerous mouse mutants with abnormal movement patterns, including the dystonia musculorum mouse (Riker et al., 1981). In the $d t$ mouse, steady-state levels of cerebellar NE appear similar to 
control levels; however, levels of the NE metabolite MHPG are elevated. In many mutants, including the staggerer, weaver, reeler, and Purkinje cell degeneration mice, elevations in cerebellar NE concentrations have been reported but appear to be a consequence of cerebellar hypoplasia (Landis et al., 1975; Kostrzewa et al., 1982; Muramoto et al., 1982). The tottering mouse, a mutant that displays focal motor seizures with a postnatal onset of 12 to 14 days, is an exception. In this mutant a hyperinnervation of the cerebellum, hippocampus, neocortex, and other areas may be due to an increase in the number of noradrenergic axons in the terminal fields of the locus ceruleus (Levitt and Nocbels, 1981). The $d t$ rat presents yet a different pattern. The rat mutant resembles the tottering mouse in demonstrating elevated cerebellar NE levels but normal cerebellar weight; however, the neocortical and hippocampal levels of NE are not reliably different from control values in the $d t$ rat. This suggests that in the $d t$ rat any hyperinnervation must involve only specific cerulear terminal fields. Our microscopic examination of the cerebellum using glyoxylic acid histochemical fluorescence does not support the hypothesis of hyperinnervation in the $d t$ rat. However, these observations must be interpreted with caution. As Kopin and others (1974) have pointed out, there are limitations on the use of fluorescence histochemistry as a quantitative technique. It may not be possible to discriminate an increase in the number of axons of the magnitude suggested by our neurochemical measurements.

Cerebellar NE is found in neurons with cell bodies in the locus ceruleus and other brainstem areas (Pickel et al., 1974; Tohyama, 1976). The NE terminals innervate somata and major dendrites of Purkinje cells (Chan-Palay, 1977). The NE innervation inhibits the firing of Purkinje cells (Bloom et al., 1971; Hoffer et al., 1971) and may play an important modulatory role in cerebellar physiology by altering the efficacy of both inhibitory and excitatory inputs to the Purkinje cells (Moises et al., 1981, 1983). Other recent work on the $d t$ rat has shown that glutamic acid decarboxylase (GAD) activity is increased selectively in deep cerebellar nuclei of the mutants in comparisons with littermate controls (Oltmans et al., 1984). A change in GAD activity at this site may indicate an alteration in the activity of the GABAergic Purkinje cells (Chan-Palay, 1982). Taken together, the findings of increased NE levels and GAD activity may reflect significant changes in cerebellar function. Further investigation will be needed to determine whether the elevated cerebellar NE seen in the $d t$ rat is causally related to either the changes in GAD activity found in the deep nuclei or to the appearance of the dystonic movements.

The absence of obvious morphological lesions of the basal ganglia has not ruled out the basal ganglia as the potential site of the primary defect in dystonia musculorum deformans. Dystonia is frequently associated with drug reactions that are presumed to involve the basal ganglia (Heline, 1978). These include complications of L-dopa therapy (Keenan, 1970) and both acute and late-onset responses to neuroleptic drugs (Crane, 1968; Burke et al., 1982). In some patients, anticholinergic and dopaminergic agents improve and cholinergic agonists and dopaminergic blockers exacerbate the symptoms of dystonia, leading some investigators to propose that dystonia is caused by a deficiency of dopamine coupled with an excess of acetylcholine (Garg, 1982; Stahl et al., 1982). However, the conclusions to be drawn about the etiology of torsion dystonia from the response of patients to drug treatments is by no means clear (Marsden, 1981), and reports of altered homovanillic acid levels in the cerebrospinal fluid of dystonic patients suggest involvement of dopaminergic systems in only certain classes of patients (Tabaddor ct al., 1978).

Measurements of DA in the dystonic rat suggest that dystonia can be present without a loss of striatal DA. However, the relative insensitivity of the $d t$ rat to the DA blocker halo- peridol seen in this study and other studies (McKeon et al., 1984) may indicate a defect in the pathway by which DA blockers exert their effects on movement. The site of such a defect is not clear at present. The apparently normal response of the $d t$ rat to apomorphine may suggest that DA receptors are not directly involved. However, the anatomical site responsible for apomorphine's effects on movement has not been precisely localized (Fink and Smith, 1980). Receptors in both the nucleus accumbens septi and the corpus striatum may be involved (Kelly et al., 1975). Thus, different populations of DA receptors may mediate the increased movement seen with apomorphine and the akinesic effects of haloperidol. Comparisons of spiroperidol binding parameters in the striatum of $d t$ and normal rats, however, do not suggest that the decreased responsiveness of the $d t$ rat to haloperidol is due to a defect at the level of the striatal DA receptor (McKeon et al., 1984).

The adequacy of the $d t$ rat as a model of human idiopathic torsion dystonia is not easily assessed. As discussed by Marsden (1976), the diagnosis of the human disease is based on the presence of clinical symptoms and the absence of other neurological deficits or probable causes. However, the availability of a rat mutant with a spontaneously occurring dystonic syndrome offers significant opportunities for testing hypotheses about the cause of dystonic symptoms. The search for biochemical markers for the disease and the evaluation of potential therapies may be facilitated. Insofar as comparisons can be made given our current scant knowledge of the human disease, the $d t$ rat appears to be a promising model for further study.

\section{References}

Altman, J., and K. Sudarshan (1975) Postnatal development of locomotion in the laboratory rat. Anim. Behav, 23: 896-920.

Askenasy, J. J., T. J. Oberman, M. Herzberg, R. Carasso, and M. Streifler (1980) Serum dopamine beta hydroxylase activity and me toclopramide provocative test in torsion dystonia. J. Neural Transm. 47: 69-77.

Baez, L. A., N. K. Eskridge, and R. Schein (1976) Postnatal develupment of dopaminergic and cholinergic catalepsy in the rat. Eur. J. Pharmacol. 36: 155-162.

Bloom, F. E., B. J. Hoffer, and G. K. Siggins (1971) Studies on norepinephrine-containing afferents to Purkinje cells of rat cerebellum. I. Localization of fibers and their synapses. Brain Res. 25: 501521.

Burke, R. E., S. Fahn, J. Jankovic, C. D. Marsden, A. E. Lang, S. Gollump, and J. Ilson (1982) Tardive dystonia: Late-onset and persistant dystonia caused by antipsychotic drugs. Neurology (NY) 32 : 1335-1346.

Chan-Palay, V. (1977) Cerebellar Dentate Nucleus: Organization, Cy tology, and Transmitters, Springer-Verlag, New York.

Chan-Palay, V. (1982) Gamma-aminobutyric acid pathways in the cerebellum studied by retrograde and anterograde transport of glutamic acid decarboxylase (GAD) antibody after in vivo injections. Prog. Brain Res. 55: 51-76.

Crane, D. E. (1968) Tardive dyskinesia in patients treated with major neuroleptics: A review of the literature. Am. J. Psychiatry 124: 4048.

de la Torre, J. C. (1980) An improved approach to histofluorescence using the SPG method for tissue monoamines. J. Neurosci. Methods 3: $1-5$.

Duchen, L. W., S. J. Strich, and D. S. Falconer (1964) Clinical and pathological studies of an hereditary neuropathy in mice (dystonia musculorum). Brain 87: 367-378.

Eldridge, R. (1970) The torsion dystonias: Literature review and genetic and clinical studies. Neurology (Minneap.) 20: 1-78.

Fahn, S., and R. Eldridge (1976) Definition of dystonia and classification of the dystonic states. Adv. Neurol. 14: 1-6.

Fink, J. S., and G. P. Smith (1980) Relationships between selective denervation of dopamine terminal fields in the anterior forebrain and behavioral responses to amphetamine and apomorphine. Brain Res. 201: 107-127.

Garg, B. P. (1982) Dystonia musculorum deformans, implications of 
therapeutic response to levodopa and carbamazepine. Arch. Neurol. 39: $376-377$.

Heline, R. D. (1978) Manual of Neurologic Therapeutics, Little, Brown and Co., Boston.

Hoffer, B. J., G. R. Siggins, and F. E. Bloom (1971) Studies on norepinephrine-containing afferents to Purkinje cells of rat cerebellum. II. Sensitivity of Purkinje cells to norepinephrine and related substances administered by microiontophoresis. Brain Res 25: 523534.

Jacobowitz, D. M., and J. S. Richardson (1978) Method for the rapid determination of norepinephrine, dopamine, and serotonin in the same brain region. Pharmacol. Biochem. Behav. 8: 515-519.

Keenan, R. E. (1970) The Eaton collaborative study of levodopa therapy in Parkinsonism: A summary. Neurology (Minneap.) 20: 46-59.

Kelly, P. H., P. W. Seviour, and S. D. Iversen (1975) Amphetamine and apomorphine responses in the rat following 6-OHDA lesions of the nucleus accumbens septi and corpus striatum. Brain Res. 94: $507-522$

Kopin, I. J., M. Palkovits, R. M. Kobayashi, and D. M. Jacobowitz (1974) Quantitative relationship of catecholamine content and histofluorescence in brain of rats. Brain Res. 80: 229-235.

Kostrzewa, R. M., C. T. IIarston, II. Fukushima, and R. Brus (1982) Noradrenergic fiber sprouting in the cerebellum. Brain Res. Bull. 9: 509-517.

Landis, S. C., W. J. Shoemaker, M. Schlumpf, and F. E. Bloom (1975) Catecholamines in mutant mouse cerebellum: Fluorescence microscopic and chemical studies. Brain Res. 93: 253-266.

Levitt, P., and J. L. Noebels (1981) Mutant mouse tottering: Selective increase of locus ceruleus axons in a defined single-locus mutation. Proc. Natl. Acad. Sci. U. S. A. 78: 4630-4634.

Lipton, S. V., J. P. McGough, and B. A. Shaywitz (1980) Effects of apomorphine on escape performance and activity in developing rat pups treated with 6-hydroxydopamine (6-OHDA). Pharmacol. Biochem. Behav. 13: 371-377.

Marsden, C. D. (1976) Dystonia: The spectrum of the disease. In The Basal Ganglia, M. D. Yahr, ed., pp. 351-367, Raven Press, New York.

Marsden, C. D. (1980) Animal models of extrapyramidal diseases. In Animal Models of Neurological Disease, F. C. Rose and P. O. Behon, eds., pp. 269-277, Pitman Medical, London.

Marsden, C. D. (1981) Treatment of torsion dystonia. In Disorders of Movement, A. Barbeau, ed., pp. 81-104, J. B. Lippincott, Philadelphia.

Marsden, C. D., and M. J. G. Harrison (1974) Idiopathic torsion dystonia (dystonia musculorum deformans). Brain 97: 793-810.

McKeon, T. W., J. F. Lorden, G. A. Oltmans, M. Beales, and S. U. Walkley (1984) Decreased catalepsy response to haloperidol in the genetically dystonic (dt) rat. Brain Res., in press.

Messer, A., and D. Gordon (1979) Changes in whole tissue biosynthesis of $\gamma$-aminobutyric acid (GABA) in basal ganglia of the dystonia $\left(d t^{\text {alh }}\right)$ mouse. Life Sci. 25: 2217-2221.

Messer, A., and N. L. Strominger (1980) An allele of the mouse mutant dystonia musculorum exhibits lesions in red nucleus and striatum. Neuroscience 5: 543-549.

Miller, M., R. Hasson, and O. Resnick (1982) Availability of phenylalanine and tyrosine for brain norepinephrine synthesis in develop- mentally protein-malnourished rats. Exp. Neurol. 77: 163-178.

Moises, H. C., R. D. Waterhouse, and D. I. Woodward (1981) I.ocus coeruleus stimulation potentiates Purkinje cell responses to afferent input: The climbing fiber system. Brain Res. 222: 43-64.

Moises, H. C., B. D. Waterhouse, and D. J. Woodward (1983) Locus coeruleus stimulation potentiates local inhibitory processes in rat cerebellum. Brain Res. Bull. 10: 795-804.

Morley, B. J., J. F. Lorden, G. B. Brown, G. E. Kemp, and R. J. Bradley (1977) Regional distribution of nicotinic acetylcholine receptors in rat brain. Brain Res. 134: 161-166.

Muramoto, O., K. Ando, and l. Kanazawa (1982) Central noradrenaline metabolism in cerebellar ataxic mice. Brain Res. 237: 387-395.

Oltmans, G. A., M. Beales, J. F. Lorden, and J. H. Gordon (1984) Alterations in cerebellar glutamic acid decarboxylase model of torsion dystonia (rat). Exp. Neurol., in press.

Pickel, V. M., M. Segal, and F. F. Bloom (1974) A radioautographic study of the efferent pathways of the nucleus locus coeruleus. J. Comp. Neurol. 155: 15-42.

Reinstein, D. K., D. McClearn, and R. L. Isaacson (1978) The development of responsiveness to dopaminergic agonists. Brain Res. 150: 216-223.

Riker, D. K., A. Messer, and R. H. Roth (1981) Increased noradrenergic metabolism in the cerebellum of the mouse dystonia musculorum. $J$. Neurochem. 37: 649-654.

Scatton, B., P. Worms, K. G. Lloyd, and G. Bartholini (1982) Cortical modulation of striatal function. Brain Res. 232: 331-343.

Shoemaker, W. J., and R. J. Wurtman (1971) Perinatal undernutrition: Accumulation of catecholamines in rat brain. Science 171: 10171019.

Stahl, S. M., K. L. Davis, and P. A. Berger (1982) The neuropharmacology of tardive dyskinesia, spontaneous dyskinesia and other dystonias. J. Clin. Psychopharmacol. 2: 321-328.

Stern, W. C., M. Miller, W. B. Forbes, P. J. Morgane, and O. Resnick (1975) Ontogeny of the levels of biogenic amines in various parts of the brain and in peripheral tissues in normal and protein malnourished rats. Exp. Neurol. 49: 314-326.

Tabaddor, K., L. I. Wolfson, and N. S. Sharpless (1978) Ventricular fluid homovanillic acid and 5 hydroxyindoleacetic acid concentrations in patients with movement disorders. Neurology (Minneap.) 28: 1249-1253.

Tohyama, M. (1976) Comparative anatomy of cerebellar catecholamine innervation from teleosts to mammals. J. Hirnforsch. 17: 43-60.

Wolfson, L. I., N. S. Sharpless, L. J. Thal, J. M. Waltz, and K. Shapiro (1983) Decreased ventricular fluid norepinephrine metabolite in childhood-onset dystonia. Neurology (NY) 33: 369-372.

Wooten, G. F., R. Eldridge, J. Axelrod, R. S. Stern (1973) Elevated plasma dopamine $\beta$-hydroxylase activity in autosomal dominant torsion dystonia. N. Engl. J. Med. 288: 284-287.

Zeigler, M. G., C. R. Lake, R. Eldridge, and I. Kopin (1976) Plasma norepinephrine and dopamine- $\beta$-hydroxylase in dystonia. Adv. Neurol. 14: 307-318.

Zeman, W. (1970) Pathology of the torsion dystonias (dystonia musculorum deformans). Neurology (Minneap.) 20: 79-88.

Zeman, W. (1976) Dystonia: An overview. Adv. Neurol. 14: 91-104. 